\title{
Chapter 12 \\ Teaching the Fundamentals of Demography: A Model-Based Approach to Fertility
}

\subsection{Introduction}

This chapter sketches some guidelines for teaching the fundamentals of demography, and gives concrete illustrations of their application to the demography of and fertility. The principles are based on: (a) over 40 years of experience in teaching demography; (b) some characteristics of a leading introductory physics text (Halliday, Resnick, and Walker 1997); and (c) recent work in the philosophy of science, notably by two representatives of the so-called semantic or model-based school, Ronald Giere $(1988,1999)$ and Nancy Cartwright $(1983,1999){ }^{1}$

The focus is on demography viewed as a science, a body of valid scientific knowledge, and on providing students of all kinds with an understanding of and ability to use this knowledge for a variety of purposes. From this perspective, the collection of demographic data by means of censuses, registers, and sample surveys, although clearly important, is seen as an ancillary activity, not part of the unique core of demographic knowledge and more a matter of applied general statistics. It is what we know about how human populations work that makes demography distinctive.

Much of our best knowledge of how populations work is to be found in the sub-area of formal demography, often labeled with the partly misleading term techniques. The argument here is that much of formal demography, while technical in some respects, can also be viewed as substantive (theoretical) knowledge. By the same token, in a model-based view of science, behavioral demography can be seen to contain, among other things, formal models whose structure is not fundamentally different from that of formal demographic models. The distinctions between

\footnotetext{
This is a slightly edited version of a paper originally published in Genus 58(2002)73-90.

${ }^{1}$ See also the stimulating suggestions for teaching introductory sociology by Stephan and Massey (1982). See Chap. 11 above.
} 
technical and substantive demography, and between formal and behavioral demography are blurred. In the model-based view of science, all models or theories are formal. $^{2}$

The guidelines for demographic teaching are meant to apply to almost any context. Fundamental principles of a science do not change depending on who is learning them. There will be differences in depth and detail, but not in the core knowledge.

I do not know whether or to what extent contemporary practice in the teaching of demography exemplifies these guidelines or the view of science on which they are based. My impression is that demographic instruction in North America does not closely correspond, and could be greatly improved. Teaching in Italy or in the French school probably comes closer to what I view as ideal. ${ }^{3}$

Support for these guidelines can be found in the practice of other, more established scientific disciplines, especially introductory courses and texts, and in the semantic or model-based school of the philosophy of science. Earlier chapters [especially Chapter 11] have discussed these rationales in some detail, so there is no need to repeat those discussions here.

\subsection{Some Concrete Examples of Abstract Fertility Models}

How might these guidelines be implemented in introducing students to the study of fertility? As a first example, let's consider the total fertility rate. This concept typically is introduced as a summary measure of age-specific fertility rates, most commonly period rates, calculated as a weighted sum (weighted by widths of age intervals) of the rates. The student may or may not have been introduced to the concept of exposure at this point. A synthetic cohort interpretation will often be used to introduce the statistical calculation, or added afterwards to give more meaning to the result - 'How many children would a woman have on average if she experienced these fertility rates?' Depending on level and context of teaching, it may be noted that the same procedure can be applied to a set of age-specific rates for a real cohort, observed over time.

This approach does not begin at the beginning. The most fundamental concept is that of a cohort of women surviving over their lifetimes and producing children. Even the distinction between real and synthetic cohort is less fundamental. And treatment of fertility behavior of a real cohort immediately raises issues such as data

\footnotetext{
${ }^{2}$ This is an unconventional view in demography, but see Keyfitz (1975).

${ }^{3}$ Book titles over the years suggest an underlying difference in attitude and approach. Lotka, writing in French, included in the title of his masterwork the phrase theorie analytique. Pressat's well-known text is called Analyse Demographique. English-language works on 'formal' demography characteristically use the words techniques or methods. The latter set of words connotes an instrumental approach, the former, notions of substantive insight.
} 
availability, incomplete experience and differential survival. The fundamental concept is that of any cohort.

An alternative approach would start with an abstract model of the lifetime fertility behavior of a cohort, and, in the manner of physics, spend a long section setting forth the basic concepts. The concepts would be represented visually to the extent possible, and used to solve several problems or carry out several 'experiments.' Initially, the only reference to data would be to a set of age-specific rates (plausible but not necessarily real) without reference to a temporal dimension other than age.

Thus:

1. Let's look at the births of a well-defined group of women (say, 1000 women at age 15) during their reproductive period, roughly ages 15 through 49 . We assume for the sake of simplicity that none dies during this period, and that they do not lack sexual partners or other means of conceiving. These are unreal assumptions, but then so is the assumption of no air-resistance or friction in beginning classical mechanics. Later we will make them more realistic. It might be worthwhile to show a graph of the number of women alive at each age, admittedly only a horizontal line. But then so is the position/time graph in a physics text showing a stationary object (zero straight-line velocity).

2. At each age, the women bear children at a given rate - births per woman in each year of age. We start with individual years of age $-15,16$, etc. Later we'll deal with 5-year age intervals. Note that for this model, the student needs only to grasp the notion of a rate as a relative number; the distinction between probability and central rate is not yet relevant since all 1000 women survive throughout. But the student is presumed to know one of the fundamental principles of demography: events $=$ rate $[s] \times$ structure (exposure, population at risk). For example: births in a given year $=$ total population times the crude birth rate, since the crude birth rate is defined as births in a given year/total population. The analogy with distance $=$ rate $\times$ time or rate $=$ distance/time is often helpful at this point.

3. The calculation of births during a given year of age is now straightforward, as is the calculation of total births over the reproductive period, and the calculation of average births per woman over the reproductive period.

4. The rates can be graphed and total births over the reproductive period viewed as the area under the curve. The typical shape of the age-specific fertility curve can be noted. It would be useful at this point, even for students without much calculus, to associate total fertility with the definite integral of the fertility-age function over all ages (or over 15-49), and to contrast this with the finite sum typically used in practice. A diagram would make the distinction clear. It also can be noted that there is no 'law of fertility,' represented by one generally accepted mathematical function (contrast Newton's law of falling bodies), although total fertility can be calculated by integration of an approximating function fit to a particular set of data (see above, Chap.7). 
5. After introducing [or assuming] the notions of weighted sum and weighted average, one can define the mean age of fertility and indicate it on the graph. The same could be done for the median age, although this measure is seldom used.

6. The basic concepts can then be modified to deal with data in 5-year intervals.

7. The notion of sex-ratio at birth can be introduced, and a calculation made of the number of daughters born as opposed to total births. This can be done overall, or, age-by-age, introducing the notion that the sex ratio at birth differs slightly (but only slightly) by age or birth order. The student is thus introduced to the idea of a one-sex population model.

At this point, the student will have learned (or reviewed) several primitive concepts in demography: relative rate; exposure; events as the product of rate [s] times population at risk; weighted sums as summary measures of rates; sums as area under an age-curve of demographic rates; areas as integrals or finite sums; 'laws' of demographic behavior and approximating functions; the sex ratio at birth; one-sex population model; 5-year versus single-year data. Note there has been no mention of period versus real cohort data, with all the complications involved.

Still, the student can do many things with the basic concepts. ${ }^{4}$ They can reverse the order of some or all the rates to convince themselves that order does not matter for total fertility, but does matter for average age. They can calculate how much difference it would make to total fertility if there were no births before age 20 or after age 35 or both. This exercise could be introduced as relevant to the frequent policy aim of eliminating teen-age fertility. ${ }^{5}$ They can calculate incomplete fertility, e.g., up to age 30 , and compare it with total fertility. These numerical exercises can be done using finite sums and, in classroom demonstrations at least, by integration of an approximating function (see Chap. 7). They can experiment with radical changes in the sex ratio at birth, such as might accompany social fads relating to gender preferences, or the consequences of China's one-child policy. In short, they can use the simple tools provided to gain greater understanding of how human reproduction works or might work.

This is demography in slow-motion and by experiment. An extremely simple abstract concept has been developed at length and in detail, and is then used to answer several substantive questions about human fertility, and provide important insights into how population reproduction works. The virtue lies not in the slowness as such, but in the fact that the student has been given a rigorous introduction to

\footnotetext{
${ }^{4}$ This assumes knowledge of how to do such calculations efficiently. Spreadsheets are useful and universally available. Mathematics programs are more powerful in the long run. Most statistical packages are not well-suited to demographic calculation unless they support programming $(R$, for example).

${ }^{5}$ Recent news stories from the United States report an increase in the average age at childbearing, partly due to a substantial reduction in teen-age fertility.
} 
basic concepts on which new and more complicated ideas can be built later in the course.

Some next steps are obvious, but note they are next steps, not first steps. The basic notions presented above can be applied to two radically different temporal contexts: the lifetime fertility experience of a real birth cohort; the cross-sectional experience in a calendar year of many different birth cohorts at different ages - the experience of a synthetic or fictitious cohort. Each would be treated in a separate section. The basic concept developed above would be seen as leading to the ordinary total fertility rate as a measure of current fertility. But it is now placed in a proper context.

This approach - developing a general concept and then applying it to two different temporal contexts (observation plans) - would by its explicitness help avoid the frequent confusion of cohort versus period data and measures. And it would be repeated, with appropriate changes, in dealing with mortality, marriage, divorce, migration, and other demographic behaviors.

The first example takes what is usually presented as a demographic measure or technique and treats it like a substantive model of demographic behavior, a summary of important knowledge of population dynamics. It is a simple model, involving unreal assumptions. But it is a good starting point.

The second example is Easterlin's socio-economic model of marital fertility, a behavioral model. It is here treated as a formally valid abstract model of fertility and fertility control. It is taken to be true 'by definition,' valuable for giving students insight into fertility decision processes, and for clarifying important behavioral issues relating to fertility. Whether or how it applies to the real world is another question. Why does fertility remain high in some developing nations? Why is it so low in Europe and North America? How might policy interventions raise or lower it? Can family planning programs help to lower fertility?

For these purposes the early, simple statement of the model (Easterlin 1975) will suffice, using equations only, or, depending on the economics background of the students, some of the indifference curves found in the original article. And again, to develop the model and present it to students, one need not have real data for all the variables. Plausible data will do. One can admit that some of the concepts and variables are difficult or even impossible to measure (for example, psychic costs of fertility control, or lifetime utility). But this does not prevent the student from gaining insight from learning and applying the model. One of the main barriers to theory development in demography has been the requirement of a one-to-one correspondence between theoretical ideas and successful, or even easy, measurement.

The Easterlin model can be summarized as follows:

\section{Definitions:}

$F=$ marital fertility [the total marital fertility rate]

$N=$ natural marital fertility, the number of births that a couple wouldhave if they did nothing to limit births 
$S=$ the probability of surviving from birth to adulthood (age 20)

$C_{n}=$ the number of surviving children a couple would have if they did nothing to limit births

$C_{d}=$ demand for children, the number of surviving children a couple wants; the number they would have if fertility control were costless

$M=$ motivation to control fertility

$R C=$ the costs of fertility control, both economic and 'psychic' costs

\section{Propositions:}

1. The level of marital fertility $\mathrm{F}$ relative to natural marital fertility $\mathrm{N}$ is a function of the extent of effective fertility control.

2. Motivation to control fertility $\mathrm{M}$ is a function of a comparison of potential surviving children $C_{n}$ and demand for children $C_{d}$. The simplest functional specification is $C_{n}-C_{d}$ but others are possible (for example, addition of a parameter, use of ratio rather than a difference).

3. The extent of effective fertility control is a function of motivation and of costs of control $R C$. Limitation will occur when motivation $>$ costs. A simple functional specification would be:

fertility control $=k[M-R C]$ if $M-R C>0$, else no fertility control; that is, fertility control is proportional to the difference between motivation and costs.

4. $C_{n}$ is a function of natural fertility $N$ and $S$, the probability of surviving from birth to adulthood, age 20: $C_{n}=N x S$.

5. $C_{d}$ is function of income, prices of children and other goods, and tastes or preferences for children - taken directly from elementary microeconomics.

6. Natural fertility $N$ is a function of coital frequency, fecundity, and fetal mortality (excluding induced abortion, which is considered a form of fertility control).

7. Fecundity is a function of many variables, notably lactation.

8. Costs of fertility control $R C$ include money costs, time and effort, and psychic costs (guilt, annoyance, fear of side effects).

As stated above, this is a relatively simple behavioral model which can be written, with suitable shorthand, on half a sheet of paper or on one panel of a classroom chalkboard. It is easily grasped by most students. A full quantitative specification is much more difficult, but again, that can come later. Even this simple model provides students with a powerful tool to help them begin thinking analytically about complex real-world problems. In my undergraduate classes, after presenting the model, I typically ask students (in discussion or exams) to use it to deal with the following questions ${ }^{6}$ : (a) Why is Canadian fertility so low? (causal analysis); (b) Do you expect it to rise appreciably over the next 10-20 years? (prediction).

\footnotetext{
${ }^{6} \mathrm{By}$ this point, the notion of overall fertility as a function of marital fertility and marriage patterns would have been presented, and the Easterlin model is presented in this context.
} 
Later, I ask them to use the same model to deal with the policy question: If the Canadian government were to decide to try to raise fertility levels, what specific measures would you advise? (policy analysis). This leads to some interesting deadends, e.g., lower motivation for fertility control by reducing natural marital fertility, or substantially increasing the costs of fertility control (this leads to a brief discussion of the infamous Rumanian case). After considering all the possibilities, students tend to focus on the notion of raising the number of children wanted $-C_{d}-$ by reducing the costs of children (notably through subsidized daycare).

The important point about the exercise is not that they reach the correct answer, if there is one, but that they have had the experience of analytic thinking about important demographic problems, using as an analytic tool a logically (if not yet quantitatively) rigorous model. There is active discussion, but it is disciplined active discussion, with the discipline supplied by the model.

\subsection{Towards More Complex Models}

The models discussed above are abstract and simple - some would say oversimplified. But so is the introductory physics of straight-line motion, or of a body falling in a vacuum under the influence of gravity. But these simple models provide an introduction to analytic thinking, as opposed to passive learning, and are the foundations of more complex models to come. A recent review of a book on migration and microevolution (Lahr 2000) includes the following as one of the book's three major conclusions: 'The frequent violation of the assumptions underlying classic population genetic models call for the development of more complex models, for which computer simulations are the main tool' (p. 2057).

Of course, more complex models will have to be developed in demography as well (Burch 1996). The assumption of no mortality in the model underlying the total fertility rate clearly is unrealistic, more so for some populations than others. Easterlin's assumption of a onetime fertility decision to maximize lifetime utility, is unrealistic in many, perhaps most, contexts. But that does not mean that one should start with these more complex models in the teaching of demography. This would be justified only if the simpler, often older, models were judged to be worthless. But they are not if they are viewed from an appropriate methodological perspective.

The simple Easterlin model can serve as a starting point for greater complexity in different ways. It can be elaborated on as a microeconomic model of fertility decision making, and there is a vast economic literature doing just that. Or it can be placed in a broader context. With the assumption that mortality or desired number of children or costs of fertility control - or any combinations of these, or other variables in the Easterlin model - are inversely related to 'development,' it can 
provide a behavioral underpinning to a model of demographic transition (cf. the biologists' development of the logistic model).

If development leads to lower mortality, given constant fertility, the number of children surviving to adulthood $\left[C_{n}\right]$ will rise, perhaps exceeding the number wanted $\left[C_{d}\right]$. By definition, this creates motivation for fertility control. Assuming $C_{d}$ also declines with development, motivation will increase even more. Unless fertility control is very costly in the broad sense defined by Easterlin, deliberate fertility control will begin and fertility will decline.

Note again that this scenario is 'true' by definition - it follows inevitably from the Easterlin model and the assumptions about relationships between development and key model variables, and it is logically coherent. Cartwright might say that it is 'true' in that it correctly captures the 'nature' of aggregate human behavior over time: we do not generally persist in accepting what we clearly do not want unless the costs of avoidance are too high. The Easterlin model, thus expanded, can be seen as a 'nomological machine,' generating the 'law' of the classic demographic transition.

Even in its simplified form - or perhaps especially in its simplified form - the Easterlin model provides students with a tool for analytic thinking about complex behavioral issues. As students work with the model to deal with various problems and exercises, it will not take long before they begin to raise questions about timing. Does the inevitable fertility decline occur immediately, in concert with mortality decline, or is there some delay or lag? Students will have no difficulty in introducing the notion of reaction time. And, depending on the level of instruction, this notion can be introduced explicitly into the model in the form of a delay.

The empirical question, as always, is whether the model can be usefully applied to one or more observed fertility transitions, whether, in Giere's words, it fits sufficiently closely in certain respects to provide an explanation, or perhaps a prediction. There is ample evidence that it does: strong empirical associations between development, mortality, and fertility; observation of the mortality/fertility lead/lag pattern in most historical transitions.

But is it the best model, in general or to explain a particular case (for example, early fertility decline in France)? Of course not, nor is it the only good model. In due time the student can be introduced to other, more complex models that emphasize culture, social interaction, wealth transfers, and other classes of variables not included or highlighted in the Easterlin model or in classic transition theory.

In short, the Easterlin model and classic transition theory are not taught as the truth about fertility decision-making and long-term transitions, but as sensible models that can serve as useful tools for the analysis of some but not all cases. Given their simplicity, they are particularly appropriate for students in the early stages of learning demography. Our physics text spends the best part of one chapter dealing with the case of motion in a straight line in the case of constant acceleration. This material is simplified, and unrealistic for almost all but laboratory settings - 
but appropriate for students to learn to understand simple systems before moving on more complex systems.

It is not that much teaching in current demography doesn't present the full panoply of models found in the literature. But it is my impression that they are often presented in fairly broad conceptual terms and passed over quickly. Older models are often presented as outmoded and of historical interest only. My suggestion is that all sensible models be taken more seriously, presented in greater detail and rigor, and then used to deal with important real-world problems.

\subsection{Concluding Comments}

There is little under the sun that is brand new. Most of the ideas outlined above can be found somewhere in the literature and practice of social science and demography. Mathematical demographers will be comfortable with the application of these ideas to formal demography. Economists will respond with a 'ho-hum' to the emphasis on teaching students to think analytically using oversimplified models, as will some sociologists who remember discussions of 'abstract analytic theory' or Lave and March's (1975) An Introduction to Models in the Social Sciences. Many social demographers will feel comfortable with the emphasis on a multiplicity of theories, with no one singled out as best.

But other ideas are apt to evoke puzzlement or resistance. One is the notion that the models of formal and behavioral demography have the same fundamental epistemological status, as abstract models of some part of the real world, 'true' by definition (assuming they are clearly and logically structured). Another, closely related, is the abandonment of logical empiricist notions of proving or disproving scientific laws. In this view, the 'underdetermination of theory by empirical data' (Turner 1987) is not some passing flaw in our science, but a central feature of all scientific knowledge, one we simply have to learn to live with and to help our students live with.

Some will wonder where this approach leaves our disciplinary penchant for multivariate analyses of census or survey data. This issue needs further study, and cannot be dealt with here. It is likely, however, that in the approach sketched above, descriptive studies using the general linear model may indeed play a smaller role, notably in teaching, giving way to more emphasis on theoretical thinking, often involving non-linear models. But statistics clearly is needed to test how closely a theoretical model fits some particular empirical case, which in any event will be described using statistics.

Historical demographers may rally against such total reliance on abstract models, preferring a more personal notion of knowledge, an 'understanding' of or 'feeling for' concrete historical cases, acquired by immersion in historical detail. Each of these and other possible objections has its merits. And not all are 
diametrically opposed to the ideas sketched above; some are complementary. But there are strong arguments for the suggested reorientation of demographic teaching. The thinking of a growing number of philosophers of science points in that direction, providing an alternative to the frustrating dead-ends of logical empiricism and its search for universal laws. Pointing in the same direction is the pedagogy of some of the strongest sciences, tried and proven in the teaching of multitudes of students that we demographers could only dream of. I have focused on physics, but other disciplines provide similar examples, notably in biology (see, for example, Gotelli 1998; for a similar approach to teaching introductory sociology, see Stephan and Massey 1982).

My suggestions are made in the context of a growing concern that demography risks losing its status as a distinct scientific field unless it pulls up its scientific specifically theoretical - socks. In many quarters, demography is seen as a purely descriptive field, the sort of descriptive work done by government statistical agencies; one often hears the terms human bookkeeping or demographic accounting. Demography is often seen as narrowly technical, concerned only with 'data grubbing.' Economists often find little in demography that they think cannot be subsumed under their discipline. In some sociological circles, demography is being surreptitiously replaced by the sociology of population, the notion being that a social gerontologist, for example, can teach demography while being innocent of the details of cohort analysis, life-table construction, or the stable population model.

My suggestions point toward a stronger demography, a discipline or sub-discipline that is unique, based on a distinctive blending of formal and behavioral models, and an unusual wealth of descriptive data. It is this discipline that we should present to our students.

\section{References}

Burch, T. K. (1996). Icons, strawmen, and precision: Reflections on demographic theories of fertility decline. The Sociological Quarterly, 37, 59-81.

Cartwright, N. D. (1983). How the laws of physics lie. Oxford: Clarendon Press.

Cartwright, N. D. (1999). The dappled world: A study of the boundaries of science. New York: Cambridge University Press.

Easterlin, R. A. (1975). An economic framework for fertility analysis. Studies in Family Planning, $6,54-63$.

Giere, R. N. (1988). Explaining science: A cognitive approach. Chicago: The University of Chicago Press.

Giere, R. N. (1999). Science without laws. Chicago: The University of Chicago Press.

Gotelli, N. J. (1998). A primer of ecology (2nd ed.). Sunderland: Sinauer Associates.

Halliday, D., Resnick, R., \& Walker, J. (1997). Fundamentals of physics (5th ed.). New York: Wiley.

Keyfitz, N. (1975). How do we know the facts of demography? Population and Development Review, 1, 267-288. 
Lahr, M.M. (2000). Wandering genes. In: Review of M. Fix, migration and colonization in human microevolution. New York: Cambridge University Press, 1999. In Science, 289, 2057.

Lave, C. A., \& March, J. G. (1975). An introduction to models in the social sciences. New York: Harper and Row.

Stephan, G. E., \& Massey, D. (1982). The undergraduate curriculum in sociology: An immodest proposal. Teaching Sociology, 9, 423-434.

Turner, S. P. (1987). Underdetermination and the promise of statistical sociology. Sociological Theory, 5, 172-184.

Open Access This chapter is licensed under the terms of the Creative Commons Attribution 4.0 International License (http://creativecommons.org/licenses/by/4.0/), which permits use, sharing, adaptation, distribution and reproduction in any medium or format, as long as you give appropriate credit to the original author(s) and the source, provide a link to the Creative Commons license and indicate if changes were made.

The images or other third party material in this chapter are included in the chapter's Creative Commons license, unless indicated otherwise in a credit line to the material. If material is not included in the chapter's Creative Commons license and your intended use is not permitted by statutory regulation or exceeds the permitted use, you will need to obtain permission directly from the copyright holder.

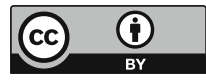

